\title{
Isolation and Characterization of Microsatellite Markers for Stenotaphrum Trin. Using 454 Sequencing Technology
}

\author{
Zhiyong Wang \\ Key Laboratory of Protection and Developmental Utilization of Tropical \\ Crop Germplasm Resources, Ministry of Education/College of Agriculture, \\ Hainan University, Haikou 570228, PR China

\section{Paul Raymer and Zhenbang Chen ${ }^{1}$ \\ Department of Crop and Soil Science, University of Georgia-Griffin Campus, Griffin, GA 30223}

Additional index words. Stenotaphrum helferi, Stenotaphrum dimidiatum, Stenotaphrum secundatum, microsatellite markers, shotgun 454 pyrosequencing

\begin{abstract}
St. augustinegrass (Stenotaphrum sp.) is a warm-season perennial turfgrass that grows widely in tropical regions around the world. St. augustinegrass is valued for both its turf performance and high levels of resistance to biotic and abiotic stresses. The current study was aimed at developing nuclear microsatellite markers for st. augustinegrass. Pyrosequencing of an enriched microsatellite library on the Roche FLX platform using a 454 Titanium kit produced 57,306 sequence reads; 2614 of which contained short tandem repeats. One hundred primer pairs were tested with 18 accessions from the U.S. Department of Agriculture National Plant Germplasm System st. augustinegrass collection grown in Griffin, GA. This collection contains both Stenotaphrum dimidiatum and Stenotaphrum secundatum accessions. Among revealed 100 primer pairs, 33 were polymorphic. A total of 175 alleles were amplified. The number of observed alleles per primer pair ranged from two to 10, with an average of 5.3. Shannon's information index and Nei's genetic diversity values were 0.4403 and 0.2873 , respectively. This set of microsatellite markers is useful for assessment of genetic diversity and construction of molecular genetic linkage maps in st. augustinegrass.
\end{abstract}

St. augustinegrass (Stenotaphrum sp.) (Poaceae) is a perennial grass native to south China. St. augustinegrass has characteristics of broad leaf blades and rapid stolon production and is widely used in home lawns, recreation parks, and sport fields. The genus, Stenotaphrum Trin., is composed of seven species, all indigenous to coastlines from East Africa to islands of the South Pacific (Busey, 1995; Sauer, 1972). The base chromosome number is $x=9$, and diploids $(2 x=$ $18)$, triploids $(3 x=27)$, tetraploids $(4 x=36)$, and hexaploids $(6 x=54)$, all exist within this genus (Milla-Lewis et al., 2013). There are two species in China, $S$. helferi Munro ex Hook. f. and Stenotaphrum subulatum Trin. These species grow on 0 to $1100 \mathrm{~m}$ altitudes and are naturally distributed in south China (Chen and Phillips, 2006; Liu, 2010). St. augustinegrass has rich genetic variation (Milla-Lewis et al., 2013; Mulkey et al., 2014); however, studies on the genetic diversity of St. Augustinegrass are limited (Busey, 1995, 2003;

Received for publication 16 Dec. 2015. Accepted for publication 11 Feb. 2016.

This research was supported by National Natural Science Foundation of China (grant no. 31560564) and the Natural Science Foundation of Hainan (no. 314067).

${ }^{1}$ Corresponding author. E-mail: zchen@uga.edu. diversity of other Stenotaphrum Trin. species ( $S$. dimidiatum and $S$. secundatum).

Molecular markers have been successfully used in classification, assessment of genetic diversity, and identification of st. augustinegrass accessions and cultivars (Milla-Lewis et al., 2013; Mulkey et al., 2014). Microsatellites or SSRs are tandemly repeated motifs of one to six bases found in the nuclear genomes of all eukaryotes and are often abundant and evenly dispersed (Ellegren, 2004; Hearnden et al., 2007; Lagercrantz et al., 1993; Tautz and Renz, 1984). SSRs have many advantages over other marker technologies including abundance, high polymorphism, codominance, easy detection, and transferability across studies. For these reasons, SSRs are frequently used to survey the population genetic diversity, construct molecular genetic linkage maps, and perform marker-assisted selection (Wang et al., 2015b). At present, there are many technologies that can be used to develop microsatellite markers. SOLiD (ABI, Norwalk, CT), 454 GS FLX (Roche, Penzberg, Germany), and Illumina Genome Analyzer (Illumina, San Diego, CA) can facilitate high-throughput genome sequencing of both noncoding and coding regions, including large-scale resequencing in wellcharacterized species or de novo transcriptome sequencing for species without reference sequences (Hearnden et al., 2007). Mulkey et al. (2014) developed 215 primer pairs of S. secundatum using an Illumina Genome Analyzer. The most common SSR methods for isolation using next generation sequencing (NGS) are transcriptome sequencing and shotgun sequencing (Wang et al., 2015a). Shotgun sequencing is an efficient option for isolating SSR markers. To take advantage of these advances, we used shotgun sequencing to isolate SSR loci rapidly and efficiently. In this study, we used Roche 454 pyrosequencing technology combined with magnetic bead enrichment FIASCO to isolate 2614 microsatellite markers for $S$. helferi. The resulting SSR sequences were characterized and validated through successful amplification of randomly selected target loci across a selection of st. augustinegrass accessions were identified and characterized using shotgun 454 pyrosequencing and used to analyze genetic

Cai et al., 2011; Cathey et al., 2011; Kinball et al., 2012; Milla-Lewis et al., 2013; Mulkey et al., 2014). Mulkey et al. $(2013,2014)$ developed simple sequence repeat markers of $S$. secundatum and identified 30,895 contigs containing simple sequence repeat (SSR) markers. In this study, microsatellite markers for $S$. helfer

Table 1. Details of sample location and inferred ploidy level from flow cytometry performed on 18 Stenotaphrum sp. accessions.

\begin{tabular}{llll}
\hline Identity & \multicolumn{1}{c}{ Species } & \multicolumn{1}{c}{ Country } & Flow cytometry-inferred ploidy \\
\hline PI 365032 & S. secundatum & South Africa & Diploid \\
PI 410353 & S. secundatum & South Africa & Diploid \\
PI 410355 & S. secundatum & South Africa & Diploid \\
PI 410361 & S. secundatum & South Africa & Diploid \\
PI 410363 & S. secundatum & South Africa & Diploid \\
PI 410364 & S. secundatum & South Africa & Diploid \\
PI 414079 & S. secundatum & United States & Diploid \\
PI 509038 & S. secundatum & Argentina & Diploid \\
PI 647924 & S. secundatum & China & Diploid \\
PI 647925 & S. secundatum & China & Diploid \\
PI 666080 & S. secundatum & United States & Diploid \\
PI 290888 & S. secundatum & South Africa & Tetraploid \\
PI 291594 & S. secundatum & Zimbabwe & Tetraploid \\
PI 300129 & S. secundatum & South Africa & Tetraploid \\
PI 300130 & S. secundatum & South Africa & Tetraploid \\
PI 671959 & S. secundatum & United States & Tetraploid \\
PI 289729 & S. dimidiatum & Madagascar & Hexaploid \\
PI 365031 & S. dimidiatum & South Africa & Hexaploid \\
\hline
\end{tabular}

${ }^{\mathrm{z}}$ Donated from this country and not originally collected from this country.

${ }^{\mathrm{y}}$ Tissue collected from University of Georgia breeding programs, Griffin, GA. 
from different geographic regions. These SSR markers provide new and valuable genomic resources for cultivar identification, assessment of genetic diversity, linkage mapping, and marker-assisted selection.

\section{Materials and Methods}

Isolation of microsatellite markers. Genomic DNA was extracted from silica geldried leaves using a plant genomic DNA Kit
(Tiangen Biotech, Beijing, China) by the manufacturer's protocol. About $1 \mu \mathrm{g}$ of genomic DNA was used to generate a shotgun library following the 454 Roche protocol. The mixtures of 3 '-biotinylated oligonucleotides $\left[(\mathrm{AG})_{10}\right.$,

Table 2. Primer sequences and characterization for microsatellite loci isolated from Stenotaphrum helferi.

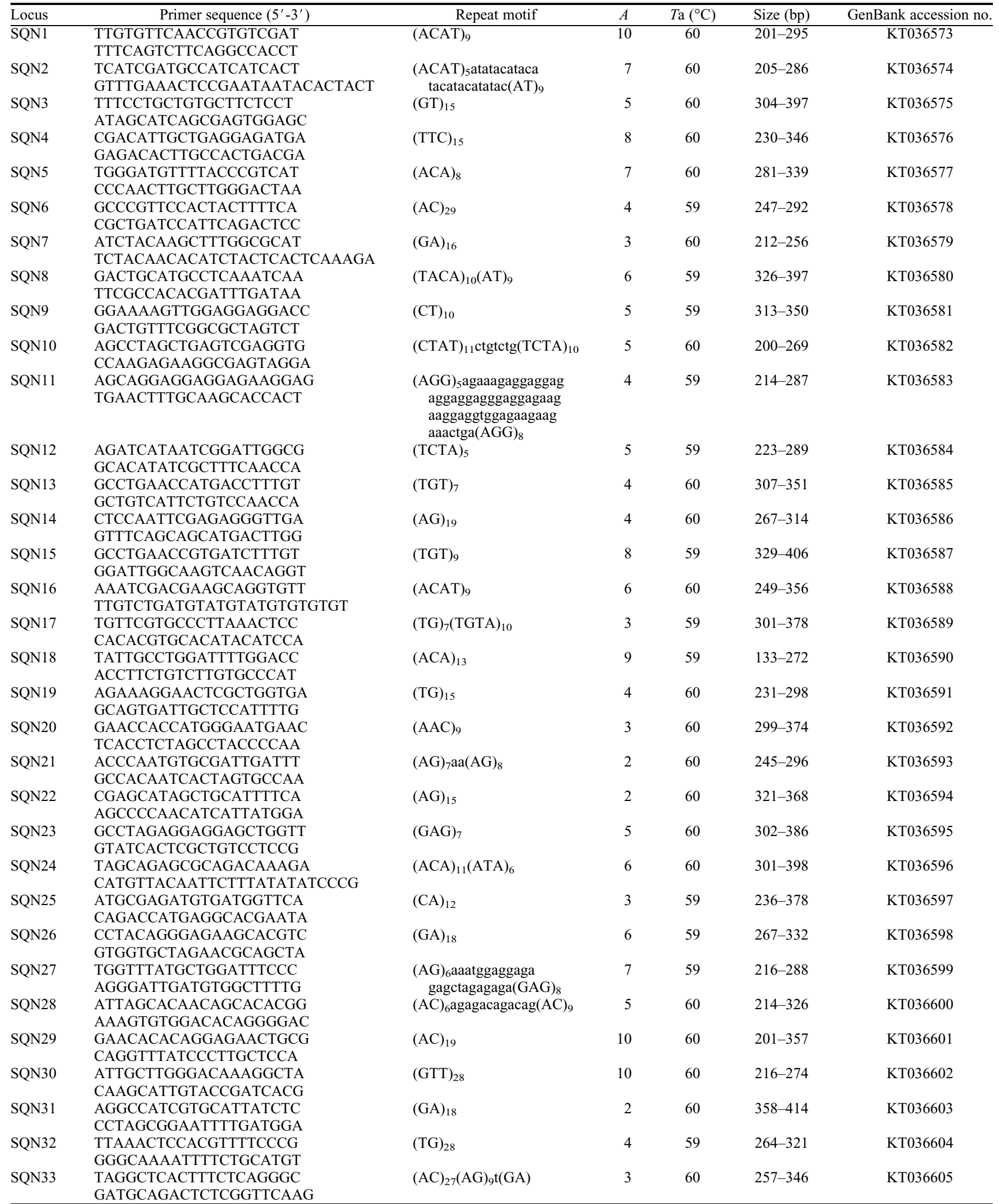

$A=$ allele number; $T \mathrm{a}=$ polymerase chain reaction annealing temperature. 
$(\mathrm{AC})_{10},(\mathrm{AAC})_{8},(\mathrm{ACG})_{8},(\mathrm{AAG})_{8},(\mathrm{ACAT}) 6$, $(\mathrm{ATCT})_{6}$, and $(\mathrm{AGG})_{8}$ ] were used to generate two separate libraries of adapter-ligated genomic DNA enriched for repetitive motifs. The enriched products were subsequently sequenced on 1/16 of a picotiter plate using a Roche 454 GS-FLX+ System (Shanghai, China). Microsatellite searching was performed using MISA (http://pgrc.ipk-gatersleben.de/misa/) with search parameters set as 10 repeat units for mononucleotides, six repeat units for dinucleotides, five repeat units for tri-, tetra-, penta-, and hexanucleotides, and the maximum interruption between two SSRs to consider a SSR as a compound set at 100 nucleotides. Primer design was conducted using Primer 3 (Rozen and Skaletsky, 2000) and the polymerase chain reaction (PCR) product size range was set at 100-400 bp. The remaining parameters were set at default values.

PCR amplification and genotyping. A total of two accessions of $S$. dimidiatum and 16 accessions of $S$. secundatum (Table 1) were used to analyze the polymorphism of the microsatellite primers. Genomic DNA was extracted from fresh leaves of each accession using the cetyltrimethylammonium bromide protocol (Murray and Thompson, 1980). The quality of the extracted DNA was verified by Thermo Scientific NanoDrop ${ }^{\mathrm{TM}}$ ND-2000c. The DNA samples were stored at $-20{ }^{\circ} \mathrm{C}$. PCR amplifications were performed in a volume of $10.0 \mu \mathrm{L}$, each containing $2.0 \mu \mathrm{L} 5 \times$ PCR buffer $(10 \mathrm{mmol} / \mathrm{L}$ Tris- $\mathrm{HCl} \mathrm{pH} 8.3,50 \mathrm{mmol} / \mathrm{L}$ $\mathrm{KCl}), 2.0 \mathrm{mmol} / \mathrm{L} \mathrm{MgCl}_{2}, 200 \mu \mathrm{mol} / \mathrm{L} \mathrm{dNTPs}$ (Invitrogen), $0.3 \mu \mathrm{mol} / \mathrm{L}$ of SSR primers, $1 \mathrm{U}$ Taq polymerase (Promega), and 100 ng template DNA using a GeneAmp PCR system 9700. Conditions used for amplification were as follows: preincubation at $95{ }^{\circ} \mathrm{C}$ for $3.0 \mathrm{~min}$; followed by 10 cycles of denaturation at $94{ }^{\circ} \mathrm{C}$ for $40 \mathrm{~s}$; annealing at $65^{\circ} \mathrm{C}$ for $45 \mathrm{~s}$; elongation at $72{ }^{\circ} \mathrm{C}$ for $1.0 \mathrm{~min}$, next followed by 30 cycles of denaturation at $94{ }^{\circ} \mathrm{C}$ for $40 \mathrm{~s}$; annealing at $55^{\circ} \mathrm{C}$ for $45 \mathrm{~s}$; elongation at $72^{\circ} \mathrm{C}$ for $1.0 \mathrm{~min}$ and a final extension step at $72{ }^{\circ} \mathrm{C}$ for $8.0 \mathrm{~min}$. A total of $9.0 \mu \mathrm{L}$ of amplified products were fractionated on a $6.0 \%$ native polyacrylamide gel against a 100-bp ladder (Promega), stained with ethidium bromide, and photographed under ultraviolet light.

Flow cytometry analysis. Flow cytometry procedures described by Milla-Lewis et al. (2013) were used to measure nuclear DNA content on the 18 accessions. Flow cytometry analyses of prepared materials were conducted on a Partec PAS (Partec, Germany) with an argon laser emitting at $488 \mathrm{~nm}$ for excitation of propidium iodide. The excised leaf material was chopped in $0.5 \mathrm{~mL}$ of an extraction buffer and the extracted leaf nuclei were stained in $2 \mathrm{~mL}$ of CyStain ${ }^{\circledR}$ PI Absolute P (Partec). The resultant solutions were filtered through $30-\mu \mathrm{m}$ nylon mesh into a $5-\mathrm{mL}$ test tube. PI 290888 and PI 365032 were used as internal standards because their nuclear contents were reported in a previous study $(1.20 \mathrm{pg} / 2 \mathrm{C})$ and should be similar to the other st. augustinegrass genotypes investigated (Milla-Lewis et al., 2013). Three measurements (three replications) were obtained for each sample. The sample DNA content was calculated as follows: sample nuclear DNA content $(\mathrm{pg} / 2 \mathrm{C})=[($ mean value of the sample peak $) /($ mean value of the internal standard)] $\times$ known nuclear DNA (1.20 pg for PI 365032).

Data analysis. Each SSR band was visually coded as present (1) or absent (0). The distance matrix and dendrogram were constructed using the Numerical Taxonomy Multivariate Analysis System (NTSYS-pc) version 2.1 (Exeter Software, Setauket, NY) software package. Genetic polymorphism (P-5\%), Nei's gene diversity $(\mathrm{He})$, and Shannon's information index $(I)$ were used to calculate Nei's standard genetic similarity coefficients (GSCs) (Nei and $\mathrm{Li}, 1979$ ) using POPGENE v.1.3.2 (Yeh et al., 2000). An unweighted pair group method with arithmetic mean (UPGMA) dendrogram was constructed within the SAHN module of the NTSYS program (Sneath and Sokal, 1973).

\section{Results and Discussion}

A total of 57,306 sequence reads were identified. Of these reads, only 2614 sequences (4.56\%) contained SSRs. Among the identified SSR loci, we randomly selected 100 sequences to design the primer pairs and to test the primer

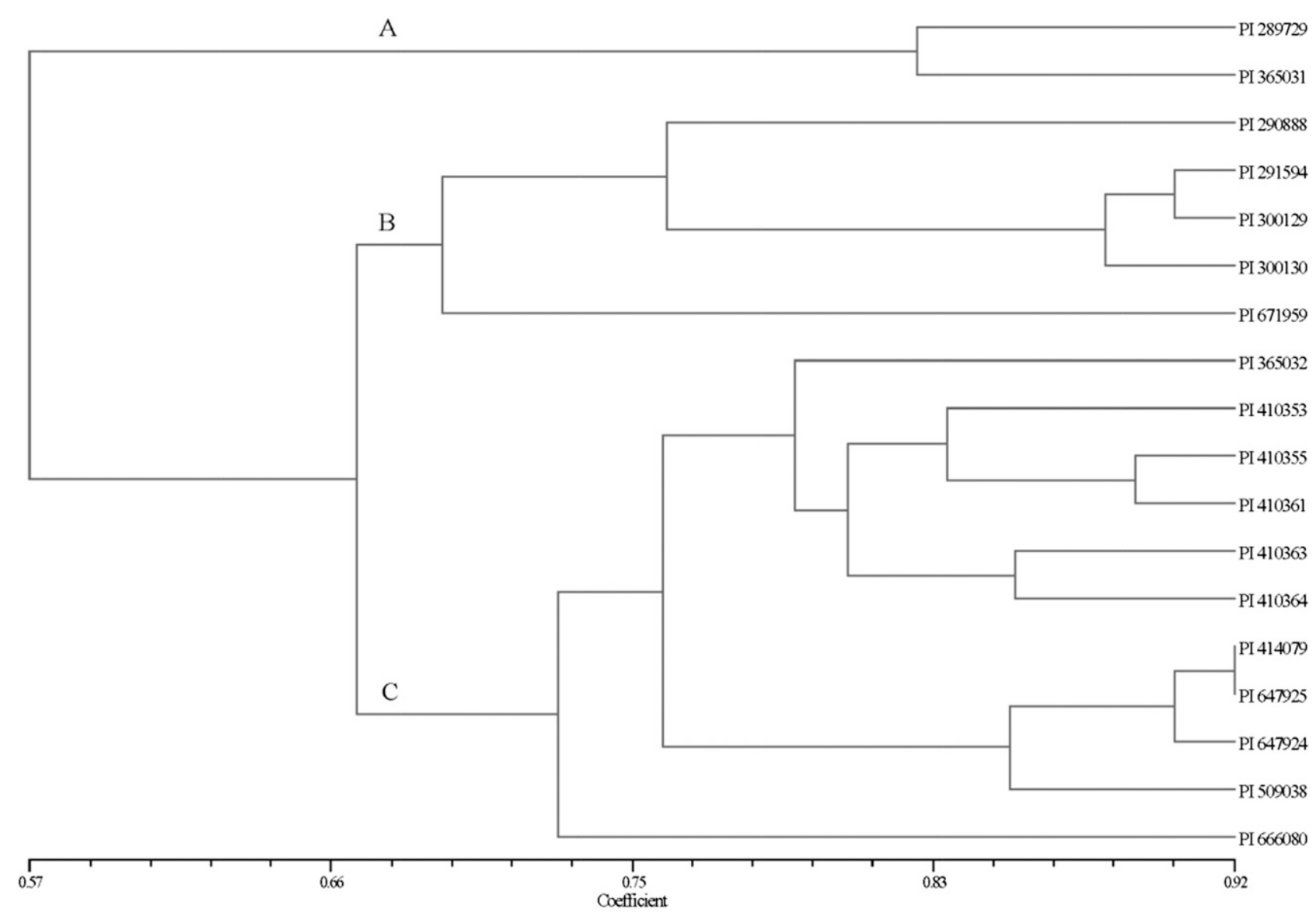

Fig. 1. Unweighted pair group method with arithmetic mean tree illustrating the genetic relationships between 18 accessions of Stenotaphrum Trin. based on genetic similarity coefficients. 
amplification efficiency. Eighty-one primer pairs were selected because of the successful amplification of target fragments, of which 33 amplified polymorphic. Sequences of these 33 primer pairs were deposited in GenBank (accession number: KT036573-KT036605) (Table 2). The number of alleles per locus ranged from two (SQN21, SQN22, and SQN31) to 10 (SQN1, SQN29, and SQN30) with an average of 5.3. Thirty-three SSR primer pairs amplified a total of 175 scorable bands, of which 161 were polymorphic (92\%). The $\mathrm{He}$ was 0.2873 and $I$ was 0.4403 on average at the species level.

A total of 161 polymorphic bands were analyzed for GSCs among the 18 st. augustinegrass accessions. The results showed that genetic diversity was relatively high among the accessions and cultivars in this study. Highly genetic diversity exists not only among three different ploidy level groups, also among different accessions within each ploidy cluster. The GSCs of the 18 accessions ranged from 0.52 to 0.92 based on the SSR data. Cluster analysis based on the GSCs, clearly classified the 18 st. augustinegrass accessions into three distinct major groups by UPGMA, which appeared to be associated with ploidy level, cluster A is associated with hexaploid accessions, cluster B with tetraploid accessions, and cluster $\mathrm{C}$ with diploid accessions (Fig. 1). High genetic diversity within each group implies that the investigated accessions are mostly natural ecotypes and not experienced major influences by human activities, such as genetic improvement in cultivar breeding. The results of this study are consistent with other published morphological and molecular studies (Busey, 1986; Milla-Lewis et al., 2013; Mulkey et al., 2013, 2014). Cluster A contained two accessions (PI 289729 and PI 365031) that are all hexaploid, which is not consistent with previous report (Milla-Lewis et al., 2013). Flow cytometry analysis showed that relative nuclear DNA content of diploid control PI 365032 and tetraploid control PI 290888 was 15.96 and 36.45 , respectively. The relative nuclear content of $1 \mathrm{C}$ can be expected ranging eight to nine. The relative nuclear DNA content of accession PI 287929 was 54.22. It was closer to the expected relative nuclear content of $6 \mathrm{C}$ (48 to 54). Cluster analysis with SSR markers also indicated that PI 289729 was hexaploid, instead of tetraploid as reported; cluster B included five st. augustinegrass accessions (PI 290888, PI 291594, PI 300129, PI 300130, and PI
671959), that are all tetraploid. The accessions from the same or nearby regions had higher GSCs and tended to cluster into the same subgroups or into neighboring subgroups. The $\mathrm{C}$ group contained 11 accessions that were all diploid. Accession PI 414079, donated from United States, shown very closely related to accessions PI 647925 and PI 647924, which were collected from China. It indicated that accession PI 414079 was possibly collected from China originally.

\section{Conclusions}

In the current study, we used shotgun 454 pyrosequencing to obtain microsatellite sequences of $S$. helferi. After thorough evaluation, we present 33 primer pairs for amplifying polymorphic microsatellite loci in Stenotaphrum sp. This first set of microsatellite markers developed for Stenotaphrum sp. using shotgun 454 pyrosequencing will be useful for the development of molecular marker-assisted breeding and the assessment of the genetic diversity of wild germplasm resources. They can also be used to construct molecular genetics linkage maps in Stenotophrum.

\section{Literature Cited}

Busey, P. 1986. Morphological identification of St. Augustinegrass cultivars. Crop Sci. 26: 28-32.

Busey, P. 1995. Genetic diversity and vulnerability of St. Augustinegrass. Crop Sci. 35:322-327.

Busey, P. 2003. St. Augustinegrass, Stenotaphrum secundatum (Walt.) Kuntze, p. 309-329. In: M.D. Casler and R.R. Duncan (eds.). Turfgrass biology, genetics, and breeding. John Wiley \& Sons, Hoboken, NJ.

Cai, X.Y., T.E. Trenholm, J. Kruse, and J.B. Sartain. 2011. Response of 'Captiva' St. Augustinegrass to shade and potassium. HortScience 46:14001403.

Cathey, S.E., J.K. Kruse, J.K. Sinclair, and M.D. Dukes. 2011. Tolerance of three warm-season turf grasses to increasing and prolonged soil water deficit. HortScience 46:1550-1555.

Chen, S.L. and S.M. Phillips. 2006. The flora of China. Science Press, Beijing, China.

Ellegren, H. 2004. Microsatellites: Simple sequences with complex evolution. Nat. Rev. Genet. 5:435445.

Hearnden, P.R., P.J. Eckermann, G.L. McMichael, M.J. Hayden, J.K. Eglinton, and K.J. Chalmers. 2007. A genetic map of 1,000 SSR and DArT markers in a wide barley cross. Theor. Appl. Genet. 115:383-391.

Kimball, J.A., M.C. Zuleta, M. Martin, K.E Kenworthy, A. Chandra, and S.R. Milla-Lewis.
2012. Assessment of molecular variation within 'Raleigh' St. Augustinegrass using AFLP markers. HortScience 47:839-844.

Lagercrantz, U., H. Ellegren, and L. Andersson. 1993. The abundance of various polymorphic microsatellite motifs differs between plants and vertebrates. Nucl. Acids Res. 21:1111-1115.

Liu, G.D. 2010. Poaceae of Hainan. Science Press, Beijing, China.

Milla-Lewis, S.R., M.C. Zuleta, G.A. Van Esbroeck, K.H. Quesenberry, and K.E. Kenworthy. 2013. Cytological and molecular characterization of genetic diversity in Stenotaphrum. Crop Sci. 53:296-308.

Mulkey, S.E., M.C. Zuleta, J.E. Keebler, J.E. Schaff, and S.R. Milla-Lewis. 2014. Development and characterization of simple sequence repeat markers for St. Augustinegrass. Crop Sci. 54:401-412.

Mulkey, S.E., M.C. Zuleta, G.A. Van Esbroeck, H.J. Lu, K.E. Kenworthy, and S.R. MillaLewis. 2013. Genetic analysis of a St. Augustinegrass germplasm collection using AFLP markers and flow cytometry. Intl. Turfgrass Soc. Res. J. 12:281-291.

Murray, M.G. and W.F. Thompson. 1980. Rapid isolation of high molecular weight plant DNA. Nucl. Acids Res. 8:4321-4325.

Nei, M. and W.H. Li. 1979. Mathematical model for studying genetic variation in terms of restriction endonucleases. Proc. Natl. Acad. Sci. USA 76:5269-5273.

Rozen, S. and H. Skaletsky. 2000. Primer3 on the WWW for general users and for biologist programmers, p. 365-386. In: S. Krawetz and S. Misener (eds.). Bioinformatics methods and protocols: Methods in molecular biology. Humana Press, Totowa, NJ.

Sauer, J.D. 1972. Revision of Stenotaphrum (Gramineae: Paniceae) with attention to its historical geography. Brittonia 24:202-222.

Sneath, P.H.A. and R.R. Sokal. 1973. Numerical taxonomy. Freeman, San Francisco, CA.

Tautz, D. and M. Renz. 1984. Simple sequences are ubiquitous repetitive components of eukaryotic genes. Nucl. Acids Res. 12:4127-4138.

Wang, X.L., Y. Li, L. Liao, C.J. Bai, and Z.Y. Wang. 2015a. Isolation and characterization of microsatellite markers for Axonopus compressus (Sw.) Beauv. (Poaceae) using 454 sequencing technology. Genet. Mol. Res. 14: 4696-4702.

Wang, X.L., L. Liao, X.Y. Zhang, C.J. Bai, and Z.Y. Wang. 2015b. Genetic diversity of Axonopus compressus (Sw.) Beauv. germplasm based on simple sequence repeat markers. HortScience 50:797-800

Yeh, F.C., R. Yang, T.J. Boyle, and J.M. Xiyan. 2000. PopGene 32. Microsoft Window-based freeware for population genetic analysis, version 1.32. Molecular Biology and Biotechnology Centre, University of Alberta, Edmonton, Canada. 